\title{
An Obstacle Detection in Order to Reduce Navigation Difficulties for Visually Impaired People
}

\author{
Tasnia Tajin Satue \\ Department of Information \& \\ Communication Technology \\ Mawlana Bhashani \\ Science and Technology University, \\ Bangladesh
}

\author{
Mohammad Badrul Alam Miah \\ Department of Information \& \\ Communication Technology \\ Mawlana Bhashani \\ Science and Technology University, \\ Bangladesh
}

\begin{abstract}
A blind person has to depend mainly on other senses, particularly of hearing ability to get information of the surroundings. Modern technology has given a blind person the ability to read through touch, but not much is available to give him or her freedom in movement except the common white stick. This paper describes the work which is based on the use of technologies to improve visually impaired people's mobility. Also,it focuses on obstacle detection in order to reduce navigation difficulties for visually impaired people. Moving through the unknown environment becomes a real challenge when can't rely on own eyes. The work present is based on the use of new technologies like Ultrasound transducer, microcontroller, and nerve stimulation to improve visually impaired people's mobility.
\end{abstract}

\section{Keywords}

Ultrasound transducer, echolocation, microcontroller, nerve stimulator.

\section{INTRODUCTION}

Visual impairment has troubled a lot in last 35 years or so. According to W.H.O, up to the June 2014; 310 million people are visually impaired in the world: 45 million blind and 265 million have low vision. About $90 \%$ of the world's visually impaired people live in developing countries. Also about $65 \%$ of all people who are visually impaired are aged 50 and older; this age group comprises about $20 \%$ of world population. 29 million children.are visually impaired out of which 1.4 million are irreversibly blind for the rest of their lives.

A blind person has to depend mainly on other senses, particularly of hearing ability to get information of the surroundings. Modern technology has given a blind person the ability to read through touch, but not much is available to give him or her freedom in movement except the common white stick. Of course, this stick is more of a flag to in for mothers rather than for the freedom of movement to the blind person him or herself. A stick allows information of the presence of obstacles over a very small range around the person and the process is also very slow and cumbersome. Unfortunately, no affordable commercial device is widely available based on modern technology to help blind people in their movement. Some attempted designs use ultrasound pulse-echo techniques, as employed by bats and dolphins, and the information is passed on to the user through sound signals delivered through headphones [1-4].

The ability of hearing is very important to a blind person as the person uses this to compensate for the loss of sight. Therefore, this method has the disadvantage that it impairs the natural hearing ability of the person through the extra stimulation coming from this new device, cluttering the normal sound. Besides, the presence of the earphone itself poses an obstruction to the free flow of sound to theirs. There for engaging the precious hearing ability to receive information through such an artificial device will rob the person of some important information that he could have otherwise obtained and deciphered. Therefore conceived of giving the information through means other than hearing.

Since dynamic obstacles usually produce noise while moving, blind people develop their sense of hearing to localize them [6]. However, they are reduced to their sense of touch when the matter is to determine where an in animate object exactly is. The common way for navigating of visionless person is using a white cane or walking cane. The walking cane is a simple and purely mechanical device dedicated to detecting static obstacles on the ground, uneven surfaces, holes and steps via simple tactile force feedback. This device is light, portable, but range is limited to its own size and it is not usable for dynamic obstacles detection neither than obstacles not located on the floor. Another option that provides the best travel aid for the blind is the guide dogs. Based on the symbiosis between the disabled owner and his dog, the training and the relationship to the animal are the keys to success.

\section{OBJECTIVE}

The basic idea is described with the help of Fig.1.1. An ultrasound pulse generated in the device will be transmitted through air and a pulse (echo) will return through reflection from an obstacle in the path. The time delay td between the transmitted and the received pulses, being twice the time taken for one way travel, will give the distance of the object from the velocity of sound in air at the particular temperature. That is, td will be larger for greater distance of object. On the receiver side, a nerve will be stimulated at a frequency $\mathrm{f}$ based on the echo time delay td.
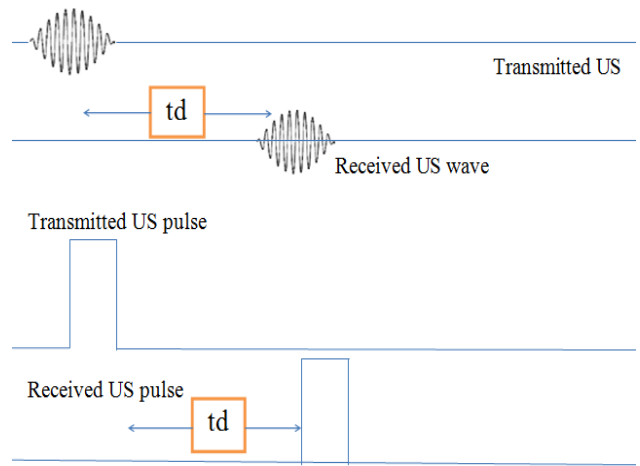

Fig 1.1: Higher frequency of stimulation for smaller time delay 
As mentioned before, an increasing stimulation frequency is desired for decreasing the distance to the object. The time delay, too, between the transmitted ultrasound wave and the received ultrasound wave, will be proportional to the distance, $\mathrm{d}$, between the obstacle and the ultrasound transducers.

The desired relationship between $\mathrm{f}$ and td is depicted in Fig.1.1 and is given by,

$$
f=f_{\max }-k \times t_{d}
$$

Where $f_{\text {max }}$ is the chosen maximum frequency of stimulation and $-k$ is the slope of the straight line. When the time delay is extremely short it should imply that the blind person is about to hit an obstacle right in front.

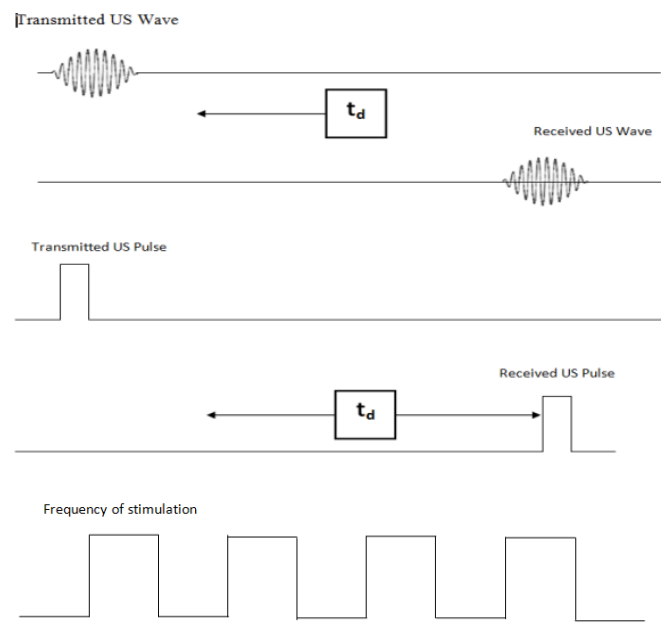

Fig 1.2: Lower frequency of stimulation for greater time delay

\section{SYSTEMS DESIGN}

The proposed design for blind aid distinctly consists of three unit

- The Obstacles Detection Unit

- Microcontroller Unit (Arduino UNO)

- Nerve Stimulator Unit

\subsection{Obstacle Detection}

The first class is based on sensory or artificial vision systems. The sensory systems emit ultrasonic or laser beams to the environment, which are reflected by the object; the system calculates the distance from the object according to the time difference between the emitted and received beam. The stereo-vision systems use the object tracking algorithms and calculate the distance by using grayscale method (VOICe). The proposed system uses ultrasonic sense or a system which basically works on the principle of the ultrasonic sound generation and alert mechanism. The system is, however, having a feedback mechanism i.e. it has an additional vibratory feedback mechanism. This enhances the overall feedback received by the blind user who receives the outputs generated in different formats of vibration i.e. high, low, medium and strong vibrations.

\subsection{Microcontroller Unit}

In this proposed unit used Arduino Uno which is a singleboard microcontroller. These systems provide sets of digital and analog pins that can be interfaced to various expansion boards ("shields") and other circuits. The boards feature serial communications interfaces, including USB on some models, for loading programs from personal computers. For programming the microcontrollers, the Arduino project provides an integrated development environment based on the Processing project, which includes support for the $\mathrm{C}$ and $\mathrm{C}++$ programming languages.

\subsection{Nerve Stimulator}

The output of the MCU goes into the nerve stimulator that includes transistors and a transformer to setup the voltage so that the current used for stimulation is below the safety limit for human nerve stimulation.[4] The frequency of nerve stimulator is equal to the frequency output from the MCU and is delivered to the user via electrodes that are connected to the surface of the skin of the blind person.

This proprosed experiment used Arduino UNO and US transducer where a US transducer has 4 pins. One is for $\mathrm{Tx}$ (transmitter ), One for Rx(receiver), another 2 pin is used for Vcc and GND . In my circuit, I used Arduino Uno pin no 6 for input, pin 7 for output and pin 13 for $\mathrm{Vcc}$, pin 12 for GND. Used pin 4 for a stimulating pulse.

\section{RESULT}

The systems were designed and implemented using Arduino and breadboard tools. The circuit was designed to produce audio feedback as well as vibration output. In cane method, whenever the obstacle is detected, a beep sound was produced using buzzer correspondingly rotation of motor gives out the vibration output. In proposed method, according to the distance of obstacle such as near, medium and far, system gives out electric shock using nerve stimulator.

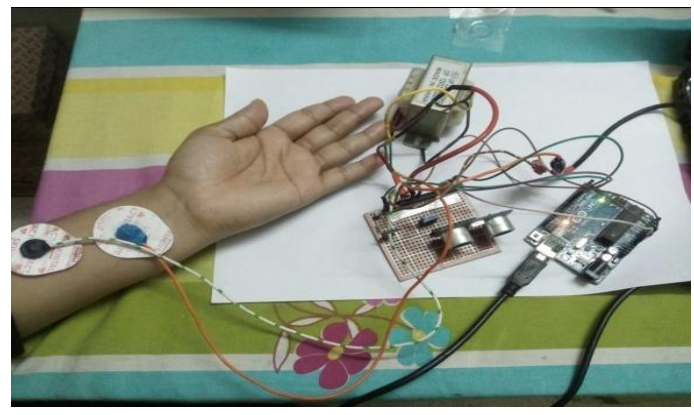

Fig 2.1: Experimental setup

Simulation Results and Numerical Analysis

\begin{tabular}{|c|c|c|}
\hline $\begin{array}{c}\text { Distance of the } \\
\text { obstacle from the } \\
\text { transmitter (cm) }\end{array}$ & $\begin{array}{c}\text { Delay time } \\
\text { between } \\
\text { transmitted and } \\
\text { received pulse } \\
(\mathbf{m s})\end{array}$ & $\begin{array}{c}\text { Stimulating } \\
\text { frequency in } \\
\text { nerve stimulator } \\
(\mathbf{H z})\end{array}$ \\
\hline 10 & 0.12 & 543 \\
\hline 20 & 0.21 & 518 \\
\hline 30 & 0.3 & 498 \\
\hline 40 & 0.4 & 479 \\
\hline 50 & 0.5 & 460 \\
\hline 60 & 0.58 & 439 \\
\hline 70 & 0.67 & 409 \\
\hline 80 & 0.78 & 389 \\
\hline 90 & 0.91 & 370 \\
\hline 100 & 1.00 & 348 \\
\hline 110 & 1.11 & 329 \\
\hline 120 & 1.23 & 312 \\
\hline 130 & 1.3 & 293 \\
\hline 140 & 1.41 & 274 \\
\hline
\end{tabular}




\begin{tabular}{|c|c|c|}
\hline 150 & 1.5 & 256 \\
\hline 160 & 1.65 & 232 \\
\hline 170 & 1.72 & 213 \\
\hline 180 & 1.85 & 189 \\
\hline 190 & 1.93 & 171 \\
\hline 200 & 2.00 & 149 \\
\hline 210 & 2.13 & 128 \\
\hline 220 & 2.21 & 107 \\
\hline 230 & 2.29 & 86 \\
\hline 240 & 2.41 & 68 \\
\hline 250 & 2.5 & 50 \\
\hline
\end{tabular}

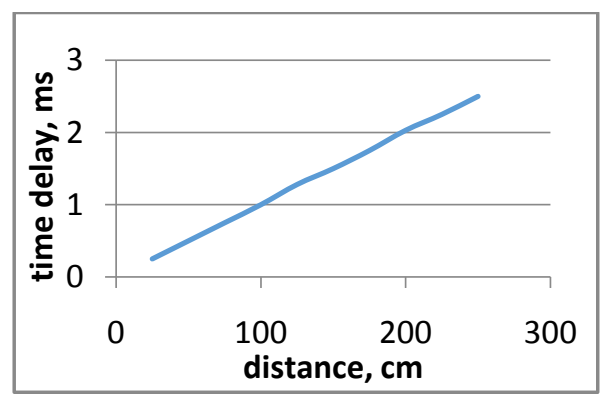

Fig 2.3: Distance vs. delay time characteristics

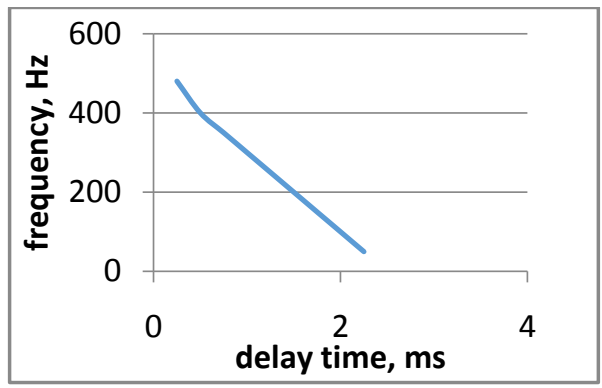

Fig 2.4: Delay time vs. frequency curve

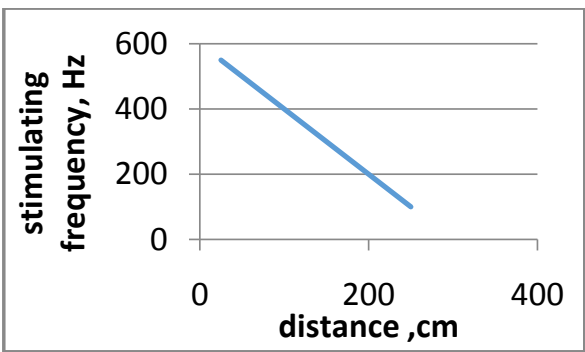

Fig 2.5: Distance vs. stimulating frequency curve

\section{CONCLUSIONS}

This new technique does not curtail the natural faculty of hearing which a blind person has, a faculty which often is enhanced to discern subtle information that a normal person cannot. The ultrasound transducers may be mounted on spectacle frame allowing the user to scan the surroundings by rotating and moving the head, thus being able to judge the size of object sin the surroundings, and the way they are moving, to some extent.

The nerve stimulations may be applied at any point where the nerve fibres run just underneath the skin. As a nearest point of stimulation one may be inclined to use the facial nerve, however, it may be rather unpleasant to the user. Other options may be the radial nerve in the upper arm, median and ulnar nerves at the elbow, or the last two nerves at the wrist. All the points except the wrist have muscle around and fatty layers under the skin. The former may cause interruption of stimulation due to muscle moving in under the electrodes, while the latter will demand much higher currents for stimulation, making it rather unpleasant and painful. Therefore, wrist appears to be the best place for stimulation having no muscle and a minimum of fat layer, although this will require a long wiring from the spectacle frame to the wrist electrodes. Placing the whole device including the transducers and electronics on the wrist is another option, however, natural movement of the hand will disturb the echo pulsing process.

This system provides a low cost alternative to the visually impaired persons in their ability to move freely in their homes without being aided. The system achieved appreciable obstacle detection above knee level and at good distance and with the attendant interface capabilities offered by the use of the microcontroller in its implementation.

Thus the present work has taken a new step to develop a useful aid for the blind which will give the person more free doming movement while avoiding obstacles and dangers.

\section{REFERENCES}

[1] WHO (2015) New estimations of visual impairment and blindness.

[2] Borenstein, J., Ulrich, I.: The GuideCane: A Computerized Travel Aid for the Active Guidance of Blind Pedestrians. In: Proceedings of the IEEE International Conference on Robotics and Automation, pp. 1283-1288 (1997).

[3] D. Dakopoulos and N. G. Bourbakis (2010) Wearable Obstacle Avoidance Electronic Travel Aids for Blind: A Survey. IEEE Trans Sys man \&Cyb, 40,1.

[4] MMuwyidUzzamanKhan,MShamiulFahadandKSiddique eRabbani, 'Ultrasoundmobilityaidforthe blind usingultra frequency modulated nerve stimulation', $6^{\text {th }}$ International Conference on Electrical \&ComputerEngineering,ICECE,Dhaka,Bangladesh.(Pap erId:p102;page:171,174;IEEECatalogNumber: CFP1068A-CDR;ISBN-978-4844-6279-7),2010.

[5] Amit Kumar M Manjutha,A.K.Majumdar,J.Mukhapadhayay and RushaPatra," An Electronic Travel Aid for Navigation of Visually Impaired Persons", $3^{\text {rd }}$ International Conference on Communication System and Networks,pp.1-5, January 2011.

[6] Kenji Terada , Minoru Fukumi and Stephen Karungaru,"Improving Mobility for Blind Persons using Video Sunglasses",pp.1-5,February 2011.

[7] TohruIfukube,TadayukiSasaki,andChenPeng, "ABlind MobilityAid Modeled After Echolocation of Bats", IEEETransactionsonbiomedicalengineering, vol.38,No.5. pp.461-465,1991 